\title{
EFFECTIVE EMBEDDED SYSTEMS SOFTWARE DESIGN METHODOLOGIES
}

\author{
T.Naga Swathi ${ }^{1}$ and M.K.Jayanthi ${ }^{2}$ \\ ${ }^{1}$ School Of Computer Science \& Engineering, VIT University, \\ Csea20@gmail.com \\ ${ }^{2}$ VIT, Vellore, Tamilnadu, INDIA \\ Jayanthi.mk@vit.ac.in
}

\begin{abstract}
This paper gives Universities needs to improve their curriculum for Technology students to meet the industry standards which will be helpful for their career. In the current improving technologies studying of embedded system is required to understand the Electronic circuits . They should include the new emerging technology such as multiprocessor system on chip where it is used in all the real time applications. In this paper design based tutorials will be discussed to understand Multiprocessor system on chip .The understanding of multiprocessor system on chip is difficult for a student and should be taught to meet the expectation from the industry. Since it is vast area, this paper proposes the most efficient tutoring method on multiprocessor system on chip.
\end{abstract}

\section{KEYWORDS}

RTOS, VLSI, MPSOC, Heterogeneous processors

\section{INTRODUCTION}

Even so many advancements has been taken place for improving the technology curriculum for engineering graduates is not been improved .There is a increasing demand for the latest technologies of the embedded systems hardware known as system on chip design as well as the software which should be an embedded C language should be taught and should be compiled using the keil software and Real Time operating systems subject should be taught in detail which is used for the real time embedded applications. .Multiprocessor system on chip design is an interesting area of embedded systems hardware design where the different applications can be built by considering various issues. In the current improving technology Embedded systems deals with real time performance, limited hardware, power consumption. An embedded system uses system on chip processors for improving performance, less resource usage and power.

\section{Multi Processor SySTeM ON CHIP}

Hardware and Software Co-design should be done at system level after the system is verified functionally the hardware and software partioning required and then simulation should be too validated by the systems per the moores law as the number of transistors increases the size should decrease and the performance of the processors is increasing drastically. so the scalability of how many processors needs to be utilized for a single application needs to be specified based on the application. Heterogeneous processor means different type of processors used

Natarajan Meghanathan, et al. (Eds): SIPM, FCST, ITCA, WSE, ACSIT, CS \& IT 06, pp. 579-584, 2012. (C) CS \& IT-CSCP 2012 
synchronously for a mobile application. Homogenous processors means same type of processor are used for parallel processing multicore applications. Where all the processors are connected parallel and increase the processing speed and decrease the execution time.

\section{CURRENT SCENARIOS}

Embedded systems programming does not have proper procedural learning. For designing of application oriented embedded system the hardware requirement will be a microcontroller surrounded by interfacing circuits like ADC and DAC, RF transmitter,ports,USART,RS232 is used to connect microcontroller with personal compiler. IIC protocol is used for interfacing different peripherals present on board. For this, a detailed knowledge of embedded $\mathrm{C}$ language is required and new library files will be added for usage of hardware circuits. Software programmes needs to interface to handle interrupts and access peripherals. Control and status registers are accessed through pointers, structures and bit fields. Programming also includes. A micro controller based approach for flash memory reprogramming this provides flexible programming of embedded firmware. This programme includes implementing the field programmability in an embedded system which use flash memory. Writing entrant code, using volatile keyword interacting with peripheral control, status registers using memory mapped Input output devices issues such as interrupt latency, watch dog timers. The programmes also covers firm ware development process including $\mathrm{C}$ start-up code, cross compilation, relocation, remote debugging.

\section{EMBEDDED SYSTEMS APPLICATIONS}

Embedded systems are used in all real time applications so the necessity of learning the hardware design is important for the engineering graduates. The main applications of embedded systems are Medical systems, Digital electronic devices, Assisted living Control systems, Automation systems, Power conservation, Weather monitoring system Robotic systems.

\section{DESIGN METHODOLOGY OF EMBEDDED HARDWARE}

For implementation of embedded projects, there are several steps involved. First step will be system specification that gives the requirements to implement the idea. The hardware design involves the necessary usage of different peripherals needs to be connected using interfacing circuit. There are two types of systems hard real time systems and soft real time systems .In hard real time systems if it crosses the time limitations it leads to catastrophe whereas soft real times which also time specific and still it works without any damage to the hardware. Once the system has been specified, the operating system loaded in to ROM.The hardware and software should be partitioned and designed in an efficient way which leads to specific application and efficient process. Software programmers must program based on the system hardware such as micro controller and different peripherals connected based on the applications. The memory requirement should also be known to be the programmer for loading the program into the limited ROM.The embedded $\mathrm{C}$ language program should always be executed in a hardware oriented platform such as KEIL MICROVISION. The microcontroller 89C51 is normally used for basic embedded applications and limited peripherals such as keypad, LCD display, stepper motor, RF transmitter, elevator, seven segment displays. The given block diagram explains basic procedure for designing embedded system. 


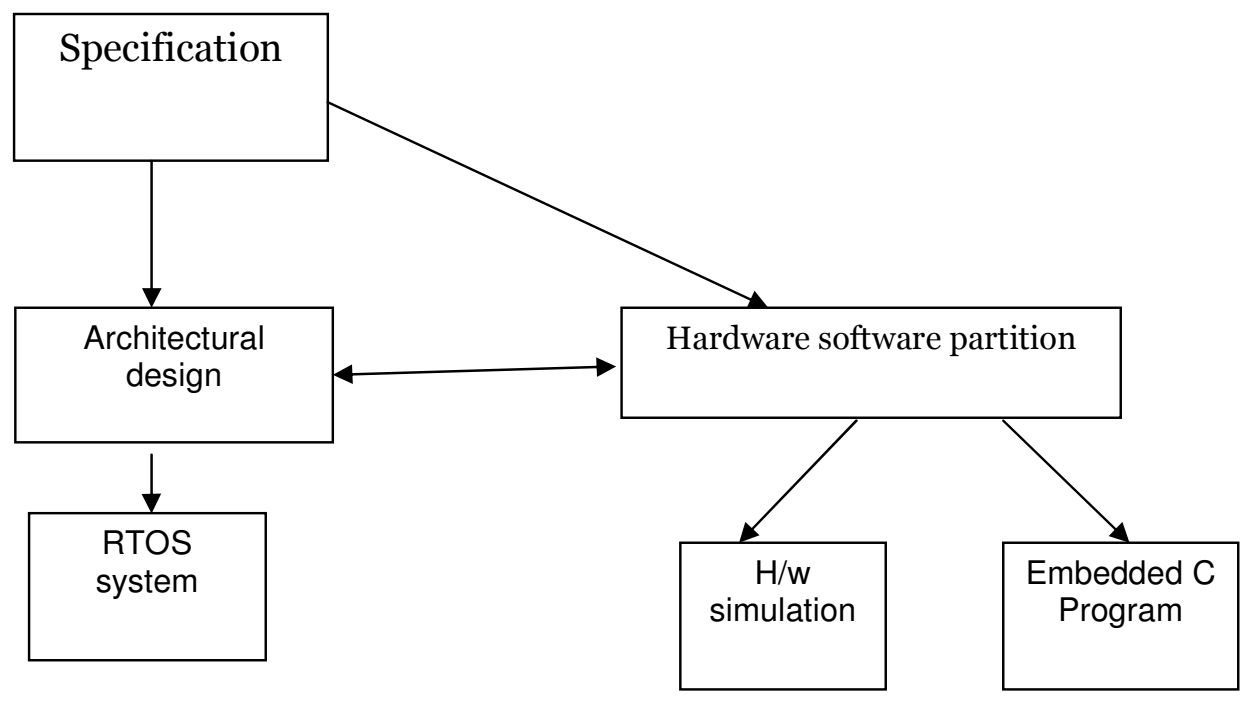

\subsection{Embedded programming}

The embedded programming is needed for designing of all the applications. Keil software is used for executing the embedded programs in efficient way. Embedded systems programmers use real time operating systems ranging from software schedulers to full featured RTOS kernels containing priority based preemptive schedulers and mechanisms for intertask communication and synchronization. This tutorial shows the features and validates the future of Real time operating systems. It requires intertask communication, synchronization mechanisms including message passing, semaphores ,message queues ,pipes ,mutexes. Dynamic memory allocation for the storage of memory also required for the real time embedded systems.

Software programmes needs to interface to handle interrupts and access peripherals. control and status registers are accessed through pointers, structures and bit fields. programming also include. A micro controller based approach for flash memory reprogramming this provides flexible programming of embedded firmware. This programmes includes implementing the field programmability in an embedded system which use flash memory. Writing entrant code, using volatile keyword interacting with peripheral control, status registers using memory mapped Input output devices issues such as interrupt latency, watch dog timers. The programmes also covers firm ware development process including $\mathrm{C}$ startup code, cross compilation, relocation, remote debugging 


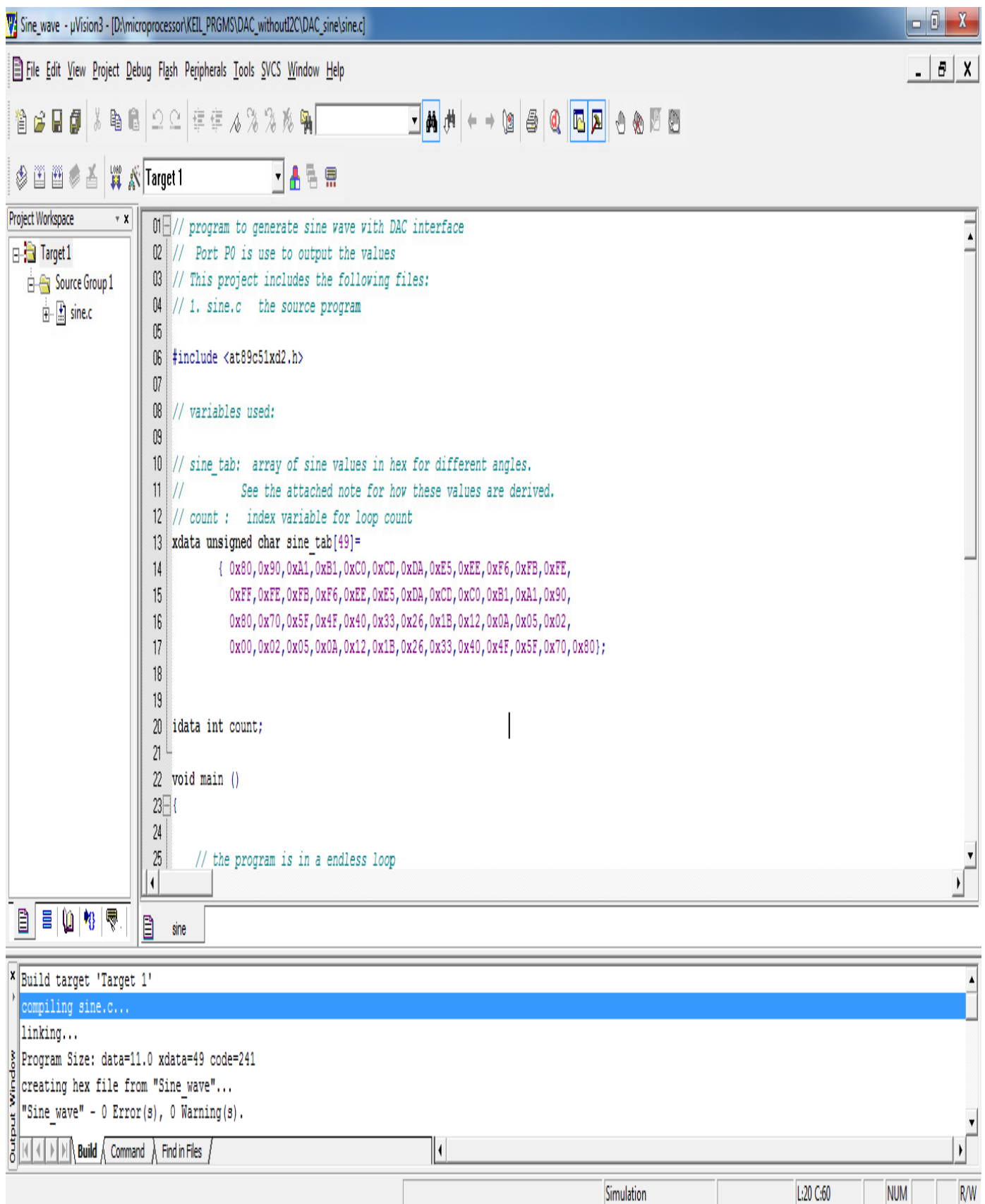


The below diagram represents the execution process and simulation of embedded systems using the $89 \mathrm{c} 51$ microcontroller in this a sine wave is been shown obtained by the software delay given using the

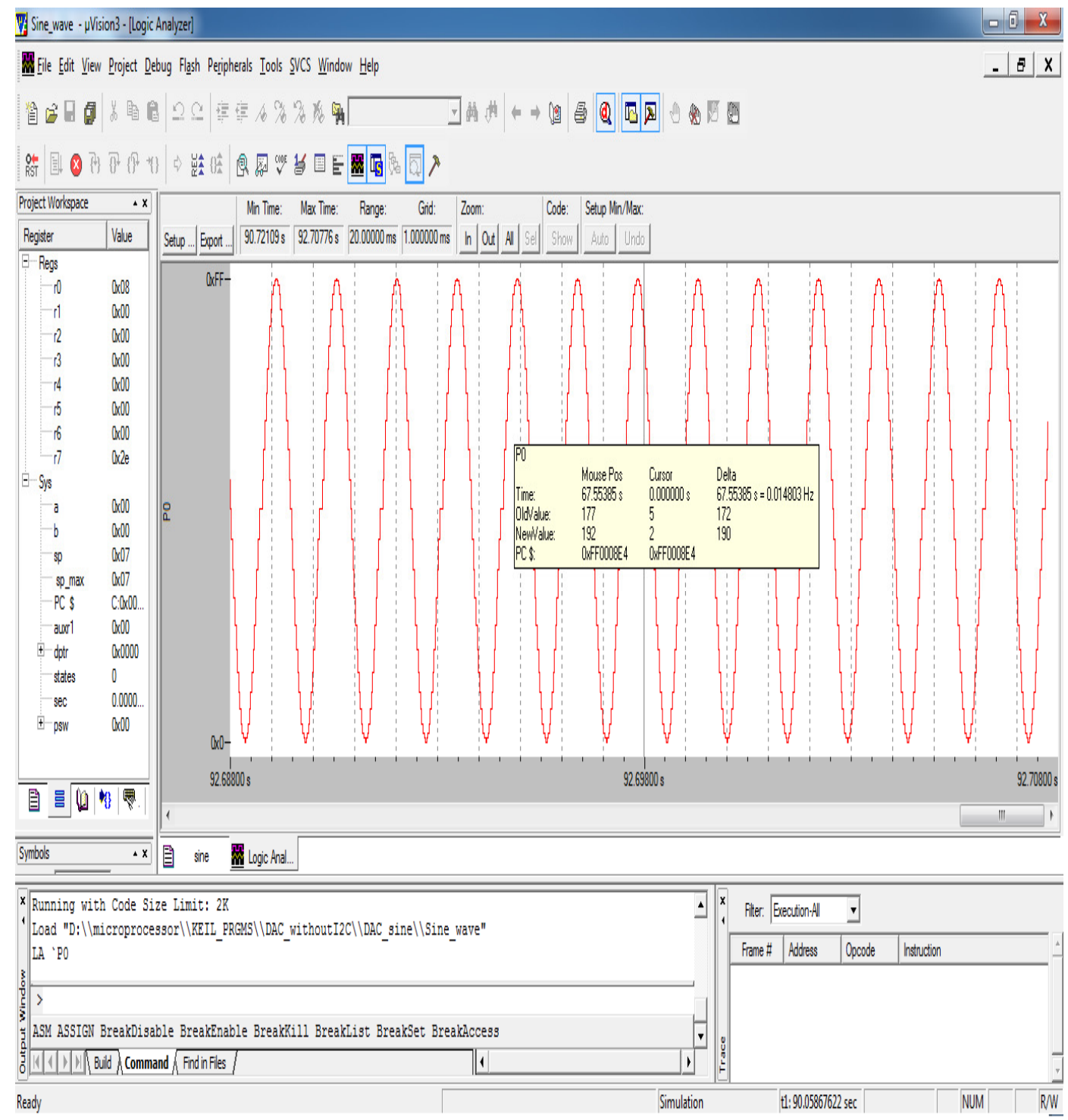

\section{Conclusions}

Embedded systems are applicable in homes, offices, hospitals, industries and consumer electronics .The applications increasing the new complexity of designing approach that emphasize on highlevel software tools and hardware/software partioning process than using the low level languages and digital logic circuits .Growing demand will increases the demand for the todays developing industrial products leading towards the programmable hardware and system software for increasing the system functionality. The real requirement of embedded systems subject needs to be added for the designing of Multiprocessor system on chip design which is used for Hardware design for engineering graduates and it meets the industry requirements. In industries there is a defienciency of skilled and well trained graduates where they work on real time projects so the 
basic subjects needs to be taught in an efficient way. There are different softwares available for the compilation of embedded c programs

\section{REFERENCES}

[1] Wolf, W.; Jerraya, A.A.; Martin, G.; , "Multiprocessor System-on-Chip (MPSoC) Technology," Computer-Aided Design of Integrated Circuits and Systems, IEEE Transactions on , vol.27, no.10, pp.1701-1713, Oct. 2008,

[2] www.coware.com

[3] www.systemc.org

[4] Title ACM Transactions on Embedded Computing Systems (TECS) TECS Homepage table of contents archive Volume 4 Issue 3, August 2005 Publisher ACM New York, NY, USA ISSN: 15399087 EISSN: $1558-3465$

[5] Martin, G. "Overview of the MPSoCs design challenge," Design Automation Conference, 2006 43rd ACM/IEEE, vol., no., pp.274-279, 0-0 0 doi: 10.1109/DAC.2006.229245

\section{Authors}

I T.Naga Swathi working as assistant professor in school of computing Science and engineering in vit university, vellore,tamilnadu

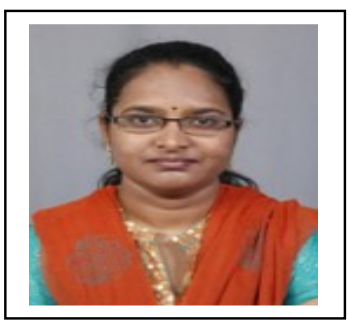

